\title{
The course of pain hypersensitivity according to painDETECT in patients with rheumatoid arthritis initiating treatment: results from the prospective FRAME-cohort study
}

Signe Rifbjerg-Madsen ${ }^{1 *}$ (D), Anton Wulf Christensen ${ }^{1}$, Mikael Boesen ${ }^{1,4}$, Robin Christensen ${ }^{1}$, Bente Danneskiold-Samsøe ${ }^{1}$, Henning Bliddal ${ }^{1}$, Lene Dreyer ${ }^{1,3}$, Henning Locht ${ }^{2}$ and Kirstine Amris ${ }^{1,2}$

\begin{abstract}
Background: Evidence is emerging that pain in rheumatoid arthritis (RA) exists without underlying inflammation. Our objective was to evaluate the prognostic value of pain classification at treatment initiation using the painDETECT questionnaire (PDQ). Outcomes were change in DAS28-CRP and RAMRIS synovitis score.

Methods: RA patients initiating a disease-modifying anti-rheumatic drug (DMARD) or initiating/ switching a biological agent were included. Follow-up time was 4 months. Clinical examination, imaging (MRI, dynamic contrast-enhanced MRI (DCE-MRI)), and patient-reported outcomes were undertaken. The PDQ was used to differentiate pain mechanisms. Mean change $(95 \% \mathrm{Cl})$ was calculated using ANCOVA. Multivariable regression models were used to determine a prognostic value.

Results: A total of 102 patients were included; 75 were enrolled for MRI. Mean changes in baseline variables were greatest in the high PDQ classification group (>18), while limited in the intermediate group (13-18). The 12 patients with high baseline PDQ score all changed pain classification group. No prognostic value of PDQ pain classification was found in relation to change of DAS28-CRP, RAMRIS score, or VAS pain. In the unadjusted model, RAMRIS score at baseline was associated with change in DAS28-CRP. The exploratory variables of DCE-MRI did not differ from other inflammatory variables.
\end{abstract}

Conclusions: In RA patients a high PDQ score (non-nociceptive pain) at baseline was not associated with worse outcomes, in fact these patients had numerically greater improvement in DAS28-CRP. However, pain classification by PDQ was not independently associated with change in DAS28-CRP, RAMRIS score, or VAS pain in the prognostic models.

Furthermore, patients classified with a high baseline PDQ score changed pain classification group. Patients with unclear pain mechanism had reduced numerically treatment response.

Trial registration: The study was approved by the Regional Ethics Committee of the Capital of Denmark April 18 2013; identification number H-3-2013-049.

Keywords: Rheumatoid arthritis, Central sensitization, painDETECT questionnaire, Prognostics, Dynamic contrastenhanced magnetic resonance imaging (DCE-MRI)

\footnotetext{
* Correspondence: signe.rifbjerg-madsen.02@regionh.dk

${ }^{1}$ The Parker Institute, Copenhagen University Hospital, Bispebjerg and

Frederiksberg, 2000 Frederiksberg, Copenhagen, Denmark

Full list of author information is available at the end of the article
}

(c) The Author(s). 2018 Open Access This article is distributed under the terms of the Creative Commons Attribution 4.0 International License (http://creativecommons.org/licenses/by/4.0/), which permits unrestricted use, distribution, and reproduction in any medium, provided you give appropriate credit to the original author(s) and the source, provide a link to the Creative Commons license, and indicate if changes were made. The Creative Commons Public Domain Dedication waiver (http://creativecommons.org/publicdomain/zero/1.0/) applies to the data made available in this article, unless otherwise stated. 


\section{Background}

Pain in rheumatoid arthritis (RA) has typically been regarded as nociceptive, that is, related to ongoing peripheral inflammation [1]. However, during the last decade, where focus has been on early diagnosis and aggressive treatment strategies in the treat-to-target regime [2], it has become clearer that in a subgroup of RA patients, pain can become an entity in its own right, probably elicited by, but not directly related to, ongoing inflammation [3]. A substantial proportion of RA patients in stable clinical remission continue to report moderate to severe pain levels [4] and studies have indicated that RA leads to widespread pain in $10-20 \%$ of patients [5]. Such observations have led to the contention that changes in the peripheral and central nervous system through processes of neural plasticity and central sensitization may play an important role [6]. As a rule, sensitization phenomena would be expected to extinguish as the tissue heals and inflammation subsides. However, a state of induced hypersensitivity of the pain system may persist in subsets of patients and lead to chronic pain states in which pain is no longer coupled to the presence of ongoing peripheral inflammation [7]. In such patients, persistent pain hypersensitivity may lead to continuous high reports of tender joints and poor global health; subcomponents of the commonly used composite disease activity score of 28 joints (DAS28-CRP) and thus overestimation of inflammatory activity. If the anti-inflammatory treatment is intensified on this background, little change in DAS28-CRP can be expected. Conversely, if inflammatory RA is left un- or not sufficiently treated it will lead to joint destruction and loss of function [8]. Identification of underlying pain mechanisms therefore has potential importance when prognosticating the effect of medical treatment on inflammation and pain.

The painDETECT questionnaire (PDQ) is a selfadministered pain classification instrument originally developed to differentiate neuropathic (non-nociceptive) from non-neuropathic (nociceptive) pain [9]. It has been increasingly used in patients with osteoarthritis and fibromyalgia to assess clinical pain features indicative of central sensitization [10-12] and has recently been introduced in studies assessing pain mechanisms in patients with RA and spondyloarthritis [13-15].

Magnetic resonance imaging (MRI) is an objective and sensitive method to assess joint inflammation. The most common scoring system in the wrist and metacarpophalangeal (MCP) joints is the OMERACT (outcome measures in rheumatoid arthritis clinical trials) RA MRI scoring (RAMRIS) system [16]. Dynamic contrast-enhanced (DCE) MRI is a technique where the sequences are acquired rapidly and sequentially before and during contrast infusion. DCEMRI has been shown to correlate better than conventional MRI with the histologic findings of synovitis $[17,18]$.
In this study, we hypothesized that a high PDQ score would serve as an indicator of central sensitization and thus a prognostic factor for a poorer treatment outcome (DAS28-CRP change) in patients with RA initiating or intensifying anti-inflammatory treatment. A possible statistical interaction between central sensitization (high PDQ score) and inflammatory load (baseline synovitis defined by hand MRI RAMRIS score) was considered as part of the hypotheses. In the exploratory part of the study, we hypothesized that DCE-MRI would capture change in inflammation and thus a possible relation to inflammatory pain mechanisms (low PDQ score) better than conventional MRI.

\section{Methods \\ Design}

The Frederiksberg Hospital's Rheumatoid Arthritis, pain assessment and Medical Evaluation (FRAME)-cohort study was conducted according to a published protocol, which contained a detailed description of the methods and prespecified analysis $[19,20]$. It was approved by the Regional Ethics Committee of the Capital of Denmark; identification number H-3-2013-049.

RA patients were recruited from departments and private clinics of rheumatology in the Copenhagen area and prospectively enrolled from March 2013 to September 2014. MRI was included in the examination program from May 2013. The examination program was conducted at Frederiksberg Hospital. Patients were assessed at treatment initiation (baseline) and after 4 months of treatment. Patients received routine care at the discretion of their rheumatologist during the trial period. Add-on of painkillers was allowed.

\section{Patients}

To be eligible, patients had to fulfil either the 1987 [21] or 2010 ACR RA criteria [8] and be $\geq 18$ years. Further, patients had to be scheduled for either (a) treatment initiation with any conventional synthetic disease-modifying antirheumatic drug (csDMARD) (patients who had not received treatment with csDMARD for at least 6 months including newly diagnosed/treatment-naïve patients) or (b) treatment initiation or change of any biologic DMARD (bDMARD).

Major exclusion criteria were intra-articular or intramuscular glucocorticoids administered less than 3 weeks prior to baseline; treatment with oral corticosteroids at doses equivalent to more than $10 \mathrm{mg}$ prednisolone/day within the 3 weeks prior to baseline; inability to pause antidepressants, anticonvulsants or other centrally acting analgesics; initiation of csDMARD therapy more than 3 weeks prior to the baseline visit (only patients initiating csDMARDs); treatment with bDMARD initiated more than 1 week prior to the baseline visit (only patients 
initiating a bDMARD). Patients who had contraindications for MRI were excluded from the MRI arm of the study. Furthermore, patients with increased risk of neuropathic pain conditions (e.g. diabetes) were excluded due to the potential to confound the pain assessment.

\section{Variables and outcome measures}

The patients underwent an examination program at baseline and follow-up, collecting information on demographics and medication and from patient-reported outcomes (PROs). Clinical examination including joint count and tender point examination conducted by the same assessor at both time points, imaging (MRI and DCE- MRI), and standard blood samples (CRP, immunoglobulin M-rheumatoid factor [IgM-RF], anti-cyclic citrullinated peptide [anti-CCP]) were also performed.

The following PROs were collected from each patient; the PDQ, the Stanford health assessment questionnaire disability index (HAQ-DI), the 36-item short form health survey (SF-36), generalized anxiety disorder assessment (GAD-10) and major depression inventory (MDI).

The PDQ is a patient-administered pain classification tool that was developed in a population of patients with various pain conditions. It has been further validated for describing pain phenotypical features in patients with inflammatory arthritis by our group [22] and is validated for use on touch screen $[9,23]$. It comprises items on pain intensity (three numeric rating scales not included in the total score), pain course patterns, pain radiation (from a pain drawing) and seven somatosensory signs and symptoms (rated on a six-category Likert scale). According to a validated algorithm, patients were assigned to one of three pain classification-groups based on a score between -1 and 38: > 18 likely neuropathic pain, 13-18 unclear pain mechanism or $<13$ likely non-neuropathic pain [9]. Several studies have used it as indicator of non-nociceptive or central pain mechanisms [10-12].

HAQ-DI is a measure of limitation of activities of daily living used for patients with RA. It assesses the patient's ability to carry out everyday tasks. It includes a visual analogue scale (VAS) evaluation of pain, fatigue and global health (GH) [24].The SF-36 assesses eight domains concerning general health, which can be summarized into a physical (PCS) and mental (MCS) component summary score. In this study the Danish version of SF36, which uses a 4-week recall period was applied [25]. The GAD-10 is a ten-item instrument developed from the Hamilton six-item anxiety scale. It measures generalized anxiety by scoring the total sum of the items [26]. The MDI is a questionnaire based on self-reported mood symptoms. It holds the ability to generate a DSM-IV and International Classification of Diseases (ICD)-10 diagnoses of major (moderate to severe) depression and to rate the severity of symptoms [27].
As standard research procedure a target hand was chosen for MRI to reflect the general level of joint inflammation. The most painful hand as reported by the patient was chosen, or, in case of no difference in pain level, the dominant hand. The examination was carried out in a $3 \mathrm{~T}$ Siemens Verio ${ }^{\circ}$ MR scanner according to a published scanning procedure [20]. Conventional coronal and axial STIR and 3D coronal T1w GRE VIBE pre- and postcontrast images were used for RAMRIS scoring. The wrist and MCP joints 2-5 were assessed according to the OMERACT RAMRIS [16, 28] and were scored for synovitis $(0-3$; total score $0-21)$ and bone marrow edema (BMO) (0-3; total score 0-58). Based on previous reports on smallest detectable difference, it was decided that the RAMRIS synovitis score had to alter by more than 1 unit to be considered a significant change [29]. All images were assessed blinded and paired by the same senior radiologist (MB).

For the explorative DCE-MRI analyses the software DYNAMIKA enterprise version 3.2.6 (http://www.ia-grp. com) was used according to a published procedure [30]. Only joints with MRI signs of inflammation ("focus joints") were included. All images were analyzed paired by the same physician (SRM). Regions of interest (ROIs) were drawn on all slices where sign of inflammation was present and collapsed into one volume of interest (VOI) for each focus joint; wrist and 2nd-5th MCP. It was decided to include tenosynovitis and capsulitis as 'signs of inflammation' in the analyses. Joints with no signal were assigned a score of 0 . Nvoxel, IRExNvoxel, MExNvoxel and IRExME were chosen as outcome measures $[17,31-35]$. The number of enhancing voxels (Nvoxel) was multiplied by the volume of each voxel in milliliter (ml) to adjust for different image sizes. The initial rate of enhancement (IRE) and maximum enhancement (ME) represent the degree of perfusion; the IRE reflects the initial rate of enhancement of the time intensity curve. ME represents the equilibrium state of the curve and reflects the amount of contrast passing into the ROI. The composite outcome measures IRExNvoxel and MExNvoxel reflect both the volume and degree of perfusion, whereas IRExME characterizes the perfusion profile of the voxels derived from the time-intensity curves.

\section{Statistical analysis}

SAS software (version 9.4, SAS Enterprise Guide 7.1, SAS Institute Inc., Cary, NC, USA) was used for all statistical analyses. PROC UNIVARIATE statement was used to summarize the data and for visual inspection of normality. Means (with standard deviations [SDs]) or medians (with interquartile ranges [IQRs]) were reported and compared by $t$ test or Kruskal-Wallis (Wilcoxon) test, respectively. Delta changes were adjusted for baseline value and compared using analysis of covariance (ANCOVA). All analyses 
were carried out according to the intention-to-treat principle, i.e. missing data at follow-up was imputed from baseline (baseline observation carried forward). A twosided $p$ value less than 0.05 was regarded as being statistically significant.

Prior to executing the FRAME-cohort study, a power calculation was performed based on the assumption that it was feasible to include $100 \mathrm{RA}$ patients during a study period of $1 \frac{1 / 2}{2}$ years [20], as no data for sample size calculation was available. Anticipating a common SD of 1.5 and the correlation between pre- and post-scores being $r=0.3$ for a paired $t$ test with a significance level of 0.05 , a sample of a 100 pairs has a power of $80 \%(0.797)$ to detect a mean change of 0.5 DAS28-CRP units. A patient population, who can expect a change of this magnitude in their disease activity, is a reasonable cohort in which to study prognostic factors of treatment response. However, this number was not reached for the MRI subsample.

The prognostic value of the PDQ score, RAMRIS score, and their interaction at baseline in relation to change of DAS28-CRP was examined by multivariable regression models using the SAS PROC GLM. As the interpretation of PDQ by nature is trichotomous, the results were expressed as least squares means per category. According to the protocol, the model was adjusted for the following prespecified confounders: age (years), sex (male/female), disease duration (month), disease activity (DAS28-CRP at baseline), group (csDMARD/bDMARD), antiCCP-positive (yes/no) and concomitant prednisolone (yes/no). Subsequently, in the fully adjusted model covariates (i.e. possible confounders) that did not contribute to the model were removed; age, antiCCP-positive (yes/no) and concomitant prednisolone (yes/no).

Secondary outcomes were change in RAMRIS synovitis score and VAS pain. Post hoc, to ensure robustness of results, a sensitivity analysis including baseline VAS pain as a confounder in the adjusted analysis of change in VAS pain was performed.

On an exploratory basis the DCE-MRI variables IRExNvoxel (in ml) or MExNvoxel (in $\mathrm{ml}$ ) for wrist were applied in the primary model examining DAS28-CRP and VAS pain change, replacing the RAMRIS synovitis score. For SRM, inter- and intra-reader agreements, intraclass correlation coefficients (ICC) (absolute agreement) for the four predefined DCE-MRI variables were tested beforehand on data from ten patients using SPSS software. Wrist and MCP joints were tested separately.

\section{Results}

Figure 1 illustrates the flow of patients. In all, 151 patients fulfilled the inclusion criteria. Of these, 48 patients were excluded. In total, 103 patients received a baseline assessment; however two were excluded post hoc. In total, 101 patients completed the PDQ at baseline; 47 initiating
csDMARD and 54 initiating bDMARD. Of these, 75 patients completed the PDQ and had an MRI scan performed at baseline and of these, 71 patients had an MRI scan performed at follow-up. This discrepancy was primarily caused by administrative delay resulting in no patients receiving MRI-scan during the first 2 months of the study period. Exclusion and reasons for dropout are further described in the figure text. Due to 3 patients not wishing to receive contrast, 72 patients with complete PDQ were included in the exploratory DCE-MRI study at baseline (not shown in Fig. 1). Of these72 patients, in all 65 completed the follow-up scan with contrast.

Baseline characteristics are described in Table 1 (some data has previously been published [36]). The distribution of patients across the PDQ classification groups were $n=66, n=23, n=12$ for PDQ score $<13,13-18$, > 18 , respectively. Statistically significant differences across the three PDQ classification groups were observed for tender joint count (TJC), tender point (TP) count, DAS28-CRP, physical function (HAQ-DI), VAS-fatigue, VAS-pain, VAS-GH, anxiety (GAD-10), depression (MDI), SF-36 PCS, and SF-36 MCS. A TP count $\geq 11$ was found for higher proportions of patients with higher PDQ classification groups; however, this was not statistically significant. No differences were found across the groups for the biochemical and imaging variables.

Changes from baseline stratified by PDQ group are presented in Table 2. Change in PDQ classification group is reported as classification consistency, i.e. number of patient that did not change classification group. Statistically significant differences in classification consistency were found for the low, intermediate, and high PDQ group; $97 \%, 83 \%$, and $0 \%$, respectively. For all clinical variables and PROs, a Ushaped curve change pattern was observed with greater change in the high and low PDQ classification group than in the intermediate. Remission at follow-up according to DAS28-CRP $(<2.6)$ was found in $41 \%, 22 \%$, and $42 \%$, respectively. The change in imaging variables did not display a distinct pattern. Statistically significant differences between the classification groups were found for delta change of CRP, DAS28-CRP, VAS GH, HAQ-DI, and SF36MCS. However for CRP, no differences between PDQ score $<13$ and $>18$, and $13-18$ and $>18$ were found. For DA28-CRP and VAS GH no differences were found between PDQ score $<13$ and PDQ score $>18$. Regarding HAQ-DI and SF36-MCS there were no difference between PDQ score $<13$ and 13-18. DCE-MRI variables are presented separately in Table 3. There were neither significant differences nor trends in the DCE-MRI variables across the groups; however, the variables IRExNvoxel (in $\mathrm{ml}$ ) and MExNvoxels (in $\mathrm{ml}$ ) for the wrist also displayed the U-shaped change pattern in line with the RAMRIS score (BME). Baseline characteristics and delta change by initiation group have previously been reported [36, 37]. 


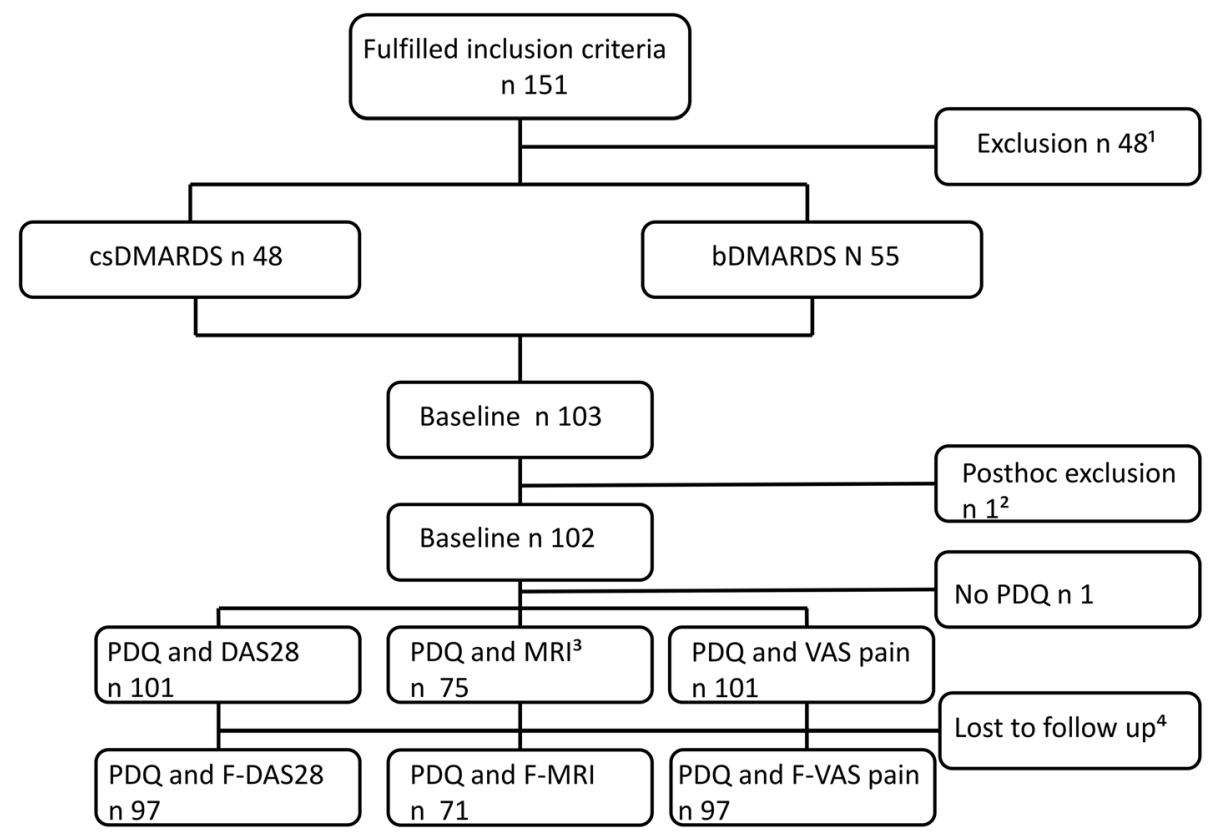

Fig. 1 Flowchart of participants. 1: Refrained from participation $(n=18)$, comorbidity with risk of neuropathic pain $(n=10)$, unable to pause antidepressants, anticonvulsants, or other centrally acting analgesics for 1 week $(n=7)$, initiated DMARD treatment $>3$ weeks ago $(n=5)$, received more than $10 \mathrm{mg}$ prednisolone < 3 weeks ago $(n=4)$, other reasons $(n=4) .2: \mathrm{Im}$. Corticosteroid between screening and baseline assessment. 3: MRI not feasible or patients declined. 4: Four patients withdrew from the study; two from each treatment group, three from the MRI subgroup. A further one patient did not participate in the follow-up MRI

In the multivariable regression models (Table 4) change in the predefined outcome variables was expressed as least square means. No interaction was found between PDQ classification group and RAMRIS synovitis neither in the protocolized analysis, nor between PDQ classification group and the DCE-MRI variables (IRExNvoxel $(\mathrm{ml})$, MExNvoxel $(\mathrm{ml})$ ) in the exploratory analysis. All interaction statements were therefore left out of the models. No prognostic value of PDQ classification was found for any of the outcomes in any of the models, sensitivity analysis included $(p=0.44)$ (data for DCE-MRI is presented as additional material, Additional file 1).

Baseline DAS28 was found to be statistically significantly associated with change in all outcomes in both adjusted models; DAS28, RAMRIS, and VAS pain $(p<0.01)$, sensitivity analysis excluded. In this analysis, baseline VAS pain was found to be significantly associated with change in VAS pain $(p<0$. 001). Baseline RAMRIS synovitis score was found to be statistically significantly positively associated with DAS28-CRP change in the unadjusted model $(p=0$. $01)$ and of RAMRIS change in both the unadjusted and the adjusted model $(p \leq 0.02)$.

In the exploratory DCE-MRI study inter and intrareader reliability showed good to excellent agreement (data is shown in Additional file 2).

\section{Discussion}

To our knowledge, this is the first study to evaluate the prognostic value of pain classification by the PDQ score in relation to change in DAS28-CRP, VAS pain, and RAMRIS score in RA patients initiating or escalating anti-inflammatory treatment. It was hypothesized that patients in the high PDQ classification group (score $>18$ ) would display constant high TJC and VAS GH as features of persistent pain hypersensitivity (central sensitization) and thus gain little or no change of DAS28-CRP following treatment initiation. In contrast however, this was not confirmed in the multiple regression analysis, we found that these patients $(n=12)$ experienced the greatest numerical change in DAS28-CRP, self-reported disease severity measures, and objective inflammatory parameters, including MRI (Table 2). This result was seen despite the fact that baseline inflammatory parameters were the same across the three PDQ classification groups, and that other baseline characteristics of the high PDQ classification group clinically could indicate presence of central sensitization (high frequency of female sex, a high number of tender joints and tender points, poor mental well-being, a high disability index, and high VAS scores).

In contrast to the patients in the low and intermediate PDQ classification -group, all patients in the high 
Table 1 Baseline characteristics stratified by PDQ group

\begin{tabular}{|c|c|c|c|c|}
\hline & $\begin{array}{l}\text { PDQ score } 7(5-9) \\
(n=66)\end{array}$ & $\begin{array}{l}\text { PDQ score } 16(13-18) \\
(n=23)\end{array}$ & $\begin{array}{l}\text { PDQ score } \geq 19 \\
(n=12)\end{array}$ & $p$ value \\
\hline PDQ score & $7(5-9)$ & $16(13-18)$ & $21(20-23)$ & $<.0001$ \\
\hline Female, n (\%) & $45(68.2)$ & $20(87.0)$ & $31(91.7)$ & 0.09 \\
\hline Age, years (SD) & $56.1(14.7)$ & $54.6(18.9)$ & $47.4(15.6)$ & 0.22 \\
\hline Initiated csDMARD, n (\%) & $37(78.7)$ & $6(12.8)$ & $4(8.5)$ & \\
\hline Disease duration, months & $15.5(1-104)$ & $53(9-47)$ & $34.5(24-149.5)$ & 0.12 \\
\hline Current smoker, n (\%) & $11(16.9)^{a}$ & $7(30.4)$ & $2(16.7)$ & 0.39 \\
\hline Corticosteroid usage, n (\%) & $11(16.7)$ & $2(8.7)$ & $3(25.0)$ & 0.42 \\
\hline 28 Swollen joint count & $2(1-7)$ & $5(2-8)$ & $2(1-4)$ & 0.09 \\
\hline 28 Tender joint count & $5(3-10)$ & $13(8-16)$ & $9.5(6.5-15)$ & $<.0001$ \\
\hline Tender point count, 0-18 & $6(4-14)$ & $10(7-16)$ & $12(6-14)$ & 0.02 \\
\hline Tender point count $\geq 11, \mathrm{n}(\%)$ & $20(30.3)$ & $11(47.8)$ & $7(58.3)$ & 0.09 \\
\hline DAS28 (SD) & $4.2(1.1)$ & $5.0(1.2)$ & $4.8(0.7)$ & $0.007+$ \\
\hline HAQ-DI, 0-3 & $0.75(0.38-1.25)$ & $1.13(0.88-1.75)$ & $1.63(1.19-1.88)$ & 0.0004 \\
\hline VAS-fatigue, mm & $53.5(27-71)$ & $75(52-89)$ & $72.5(60.5-87)$ & 0.002 \\
\hline VAS-pain, mm & $42(24-60)$ & $69(50-82)$ & $63(45.5-80.5)$ & 0.0003 \\
\hline VAS-global health, mm & $56(32-77)$ & $73(46-85)$ & $78(61.5-90)$ & $0.03 \neq$ \\
\hline GAD-10 score, 0-50 & $6(3-10)^{a}$ & $10(6-17)$ & $9(9-12)$ & 0.008 \\
\hline MDI score, 0-50 & $8(4-12)^{a}$ & $11(6-25)$ & $13.5(9-19.5)$ & 0.01 \\
\hline SF-36 PCS, 0-100 & $35(29-42)$ & $33(26-37)$ & $28(24-32)$ & $0.02 \ddagger$ \\
\hline SF-36 MCS, 0-100 & $51(40-57)$ & $43(31-51)$ & $37(31-49)$ & 0.005 \\
\hline $\mathrm{CRP}, \mathrm{mg} / \mathrm{mL}$ & $8(3-15)$ & $4(0.5-19)$ & $3(0.8-8.5)$ & 0.13 \\
\hline IgM-RF positive, n (\%) & $41(62.1)$ & $15(65.2)$ & $8(66.7)$ & 1.0 \\
\hline Anti-CCP positive, n (\%) & $46(70)$ & $15(65)$ & $7(58)$ & 0.72 \\
\hline RAMRIS hand synovitis & $7(5-10)$ & $9.5(7-11)$ & $7(6-9)$ & 0.18 \\
\hline RAMRIS hand edema & $5(1-10)$ & $9.5(3-22)$ & $5(2-13)$ & 0.12 \\
\hline
\end{tabular}

Values are median (25th, 75th percentiles) unless specified otherwise

Unless specified otherwise significant $p$ values reflect difference between PDQ score $<13$ and $13-18$ and $\geq 19$, while there is no difference between PDQ score $13-18$ and $\geq 19$

${ }^{\dagger}$ Only difference between PDQ score $<13$ and $13-18$

${ }^{\ddagger}$ Only difference between PDQ score $<13$ and $\geq 19$

RAMRIS hand; wrist+MCP scores

One patient receiving MRI had no corresponding PDQ score. 26 patients did not receive MRI scan. Number of patients (n) for RAMRIS parameters: PDQ score < 13, $n=51$; PDQ score 13-18, $n=18$; PDQ score $>18, n=6$. (a) One missing observation. (b) Three missing observations

PDQ classification group changed pain classification following treatment initiation or escalation. These findings could indicate that in these particular patients, a high PDQ score might not have marked central pain mechanisms uncoupled to ongoing inflammation, but could have reflected reversible inflammatory driven pain hypersensitivity, i.e. normal neuroplasticity. However, regression toward the mean needs to be taken into account. Interestingly, the observed numerically changes of the variables were smallest in the intermediate PDQ group (score 13-18), total hand RAMRIS synovitis score excluded. Thus, patients with an unclear pain mechanistic background had the poorest response to medical treatment also reflected in the U-shaped DAS28 remission pattern across the PDQ classification groups, indicating that the uncoupling of pain mechanism from present inflammation could be found in this group.

In the multivariable regression analyses, the PDQ did not have prognostic value in relation to change of DAS28CRP, RAMRIS score, or VAS pain. No interaction between PDQ score and baseline RAMRIS synovitis score was found in any of the regression analyses including the exploratory DCE-MRI analyses indicating no relation between ongoing inflammation and pain phenotype (pain classification group). However, in the light of the relatively small sub-sample of patients with a high PDQ score at baseline, it does not seem reasonable to reject any prognostic value of the PDQ at this point. 
Table 2 Mean change stratified by baseline PDQ group adjusted for baseline value

\begin{tabular}{|c|c|c|c|c|}
\hline & $\begin{array}{l}\text { PDQ score }<13 \\
(n=66)\end{array}$ & $\begin{array}{l}\text { PDQ score } 13-18 \\
(n=23)\end{array}$ & $\begin{array}{l}\text { PDQ score } \geq 19 \\
(n=12)\end{array}$ & $p$ value \\
\hline PDQ consistency, N (\%) & $64(97)$ & $19(83)$ & $0(0)$ & $<0.001^{\dagger}$ \\
\hline$\Delta \mathrm{PDQ}$ score & $-4.10(-5.74 ;-2.45)$ & $-2.20(-4.64 ; 0.25)$ & $-5.49(-9.97 ;-1.02)$ & 0.08 \\
\hline$\Delta 28 \mathrm{SJC}$ & $-2.71(-3.26 ;-2.17)$ & $-2.27(-3.21 ;-1.34)$ & $-2.48(-3.77 ;-1.19)$ & 0.71 \\
\hline$\Delta 28 \mathrm{TJC}$ & $-4.63(-5.67 ;-3.60)$ & $-4.36(-5.14 ;-1.58)$ & $-5.57(-7.96 ;-3.18)$ & 0.28 \\
\hline$\Delta C R P, \mathrm{mg} / \mathrm{mL}$ & $-6.93(-8.54 ;-5.32)$ & $-2.81(-5.54 ;-0.08)$ & $-6.28(-10.10 ;-2.47)$ & $0.04 a$ \\
\hline$\triangle \mathrm{DAS28-CRP}$ & $-1.47(-1.74 ;-1.20)$ & $-0.8(-1.27 ;-0.34)$ & $-1.62(-2.25 ;-1.00)$ & $0.03 b$ \\
\hline$\Delta$ Tender point count & $-2.70(-3.87 ;-1.57)$ & $-1.56(-3.48 ; 0.36)$ & $-3.83(-6.46 ;-1.20)$ & 0.35 \\
\hline$\Delta$ VAS-fatigue & $-18.31(-25.55 ;-13.06)$ & $-8.19(-17.07 ; 0.68)$ & $-22.20(-34.48 ;-9.91)$ & 0.09 \\
\hline$\Delta$ VAS-pain & $-23.27(-28.21 ;-18.33)$ & $-12.99(-21.40 ;-4.57)$ & $-26.86(-38.34 ;-15.38)$ & 0.07 \\
\hline$\Delta$ VAS-global health & $-26.20(-31.15 ;-21.25)$ & $-13.49(-21.83 ;-5.15)$ & $-31.72(-43.44 ;-20.01)$ & $0.01 b$ \\
\hline$\Delta \mathrm{HAQ}-\mathrm{DI}$ & $-0.34(-0.44 ;-0.24)$ & $-0.15(-0.32 ; 0.02)$ & $-0.74(-0.98 ;-0.50)$ & $<0.001 c$ \\
\hline$\Delta \mathrm{MDI}$-total & $-3.04(-4.39 ;-1.68)$ & $-2.54(-4.83 ;-0.25)$ & $-3.68(-6.80 ;-0.55)$ & 0.84 \\
\hline$\Delta$ GAD10-total & $-2.24(-3.18 ;-1.30)$ & $-1.56(-3.15 ; 0.02)$ & $-2.10(-4.27 ; 0.07)$ & 0.76 \\
\hline$\Delta$ SF36-MCS & $5.5(3.34 ; 7.60)$ & $3.70(0.09 ; 7.31)$ & $11.38(6.40 ; 16.36)$ & $0.04 c$ \\
\hline$\Delta$ SF36-PCS & $6.47(4.39 ; 8.56)$ & $4.94(1.43 ; 8.44)$ & $8.96(3.97 ; 13.94)$ & 0.42 \\
\hline$\Delta$ RAMRIS H synovitis & $-1.69(-2.32 ;-1.07)$ & $-1.81(-2.90,-0.71)$ & $-2.47(-4.28 ;-0.66)$ & 0.72 \\
\hline$\Delta$ RAMRIS H edema & $-2.17(-4.00 ;-0.34)$ & $-0.57(-3.80 ; 2.66)$ & $-3.46(-8.72 ; 1.81)$ & 0.58 \\
\hline
\end{tabular}

Values are means $(95 \% \mathrm{Cl})$. Negative numbers indicate improvement. ANCOVA (BOCF) was used for the analyses unless otherwise indicated. $+\mathrm{Chi}$-square test RAMRIS $H$ hand $(\mathrm{W}+\mathrm{M})$. $W$ wrist. $M$ MCP joints

(n) for RAMRIS parameters: PDQ score $<13 ; n=51$, PDQ score 13-18; $n=18$, PDQ score $>18 ; n=6$. (a) No difference between PDQ score $<13$ and $>18$, and $13-18$ and $>18$. (b) No difference between PDQ score $<13$ and PDQ score $>18$. (c) No difference between PDQ score $<13$ and $13-18$

Table 3 Baseline values and mean changes (adjusted for baseline value) for the exploratory DCE-MRI variables

\begin{tabular}{|c|c|c|c|c|}
\hline & PDQ score $<13$ & PDQ score 13-18 & PDQ score $\geq 19$ & $p$ value \\
\hline \multicolumn{5}{|l|}{ Baseline values } \\
\hline Nvoxel in ml wrist & $13.17(5.53-29.40)$ & $14.78(7.22-25.84)$ & $14.21(8.28-22.70)$ & 0.93 \\
\hline Nvoxel in ml MCP & $1.65(0.47-6.57)$ & $3.12(0.80-9.22)$ & $1.69(0.00-3.91)$ & 0.56 \\
\hline IRE $\times$ Nvoxel in ml wrist & $0.15(0.05-0.67)$ & $0.25(0.04-0.67)$ & $0.19(0.06-0.56)$ & 0.91 \\
\hline IRE $\times$ Nvoxel in $\mathrm{ml} \mathrm{MCP}$ & $0.02(0.002-0.15)$ & $0.06(0.003-0.24)$ & $0.006(0.00-0.07)$ & 0.31 \\
\hline ME $\times$ Nvoxel in $\mathrm{ml}$ wrist & $21.43(9.76-62.29)$ & $26.65(10.09-56.40)$ & $26.73(14.72-42.67)$ & 0.92 \\
\hline ME $\times$ Nvoxel in $\mathrm{ml}$ MCP & $2.30(0.67-14.23)$ & $6.70(0.91-21.34)$ & $2.46(0.00-7.42)$ & 0.50 \\
\hline IRE $\times$ ME wrist & $0.02(0.01-0.05)$ & $0.03(0.01-0.06)$ & $0.02(0.01-0.05)$ & 0.87 \\
\hline IRE $\times$ ME MCP & $0.03(0.01-0.09)$ & $0.04(0.01-0.12)$ & $0.01(0.00-0.05)$ & 0.21 \\
\hline \multicolumn{5}{|l|}{ Mean changes } \\
\hline$\Delta$ Nvoxel in ml wrist & $-2.22(-4.84 ; 0.41)$ & $-2.47(-6.93 ; 1.99)$ & $-2.87(-10.37 ; 4.63)$ & 0.98 \\
\hline$\Delta$ Nvoxel in $\mathrm{ml}$ MCP & $-1.99(-3.02 ;-0.96)$ & $-1.72(-3.47 ; 0.03)$ & $-0.79(-3.74 ; 2.17)$ & 0.74 \\
\hline$\Delta$ IRE $\times$ Nvoxel in $\mathrm{ml}$ wrist & $-0.18(-0.28 ;-0.07)$ & $-0.11(-0.29 ; 0.07)$ & $-0.17(-0.46 ; 0.13)$ & 0.80 \\
\hline$\Delta$ IRE $\times$ Nvoxel in $\mathrm{ml}$ MCP & $-0.07(-0.11 ;-0.03)$ & $-0.06(-0.13 ; 0.003)$ & $-0.06(-0.17 ; 0.06)$ & 0.99 \\
\hline$\Delta \mathrm{ME} \times \mathrm{N}$ voxel in $\mathrm{ml}$ wrist & $-7.72(-14.69 ;-0.76)$ & $-5.55(-17.37 ; 6.27)$ & $-7.90(-27.80 ; 12.00)$ & 0.95 \\
\hline$\triangle \mathrm{ME} \times \mathrm{N}$ voxel in $\mathrm{ml} \mathrm{MCP}$ & $-5.17(-7.42 ;-2.92)$ & $-4.82(-8.64 ;-1.00)$ & $-3.12(-9.58 ; 3.33)$ & 0.84 \\
\hline$\Delta \mathrm{IRE} \times \mathrm{ME}$ wrist & $-0.01(-0.02 ;-0.01)$ & $-0.01(-0.02 ; 0.003)$ & $-0.01(-0.03 ; 0.01)$ & 0.81 \\
\hline$\Delta \mathrm{IRE} \times \mathrm{ME} \mathrm{MCP}$ & $-0.03(-0.04 ;-0.02)$ & $-0.03(-0.06 ;-0.01)$ & $-0.03(-0.07 ; 0.01)$ & 0.96 \\
\hline
\end{tabular}


Table 4 Multivariable regression models examining change expressed as least squares means (95\% Cl)

\begin{tabular}{|c|c|c|c|c|}
\hline Protocolized models & PDQ score $<13$ & PDQ score 13-18 & PDQ score > 18 & $p$ value \\
\hline \multicolumn{5}{|l|}{ Unadjusted model } \\
\hline$\triangle \mathrm{DAS} 28$ & $-1.30(-1.64 ;-0.97)$ & $-1.17(-1.74 ;-0.60)$ & $-2.08(-3.06 ;-1.10)$ & 0.27 \\
\hline$\triangle$ RAMRIS Synovitis & $-1.68(-2.30 ;-1.06)$ & $-1.75(-2.80 ;-0.70)$ & $-2.46(-4.25 ;-0.66)$ & 0.72 \\
\hline$\triangle \mathrm{VAS}$ pain & $-17.57(-24.13 ;-11.02)$ & $-25.45(-36.56 ;-14.33)$ & $-38.13(-57.15 ;-19.11)$ & 0.09 \\
\hline \multicolumn{5}{|l|}{ Adjusted } \\
\hline$\triangle \mathrm{DAS} 28$ & $-1.49(-1.81 ;-1.18)$ & $-1.16(-1.70 ;-0.61)$ & $-2.06(-2.94 ;-1.17)$ & 0.17 \\
\hline$\triangle$ RAMRIS Synovitis & $-1.78(-2.44 ;-1.26)$ & $-1.72(-2.86 ;-0.57)$ & $-2.48(-4.34 ;-0.63)$ & 0.74 \\
\hline$\triangle \mathrm{VAS}$ pain & $-19.36(-25.89 ;-12.83)$ & $-24.71(-36.07 ;-13.35)$ & $-38.04(-56.50 ;-19.56)$ & 0.15 \\
\hline
\end{tabular}

No interaction was found in any of the models. The protocolized unadjusted models included the baseline PDQ classification groups as a trichotomous variable and RAMRIS synovitis score for the hand. The adjusted models further included female sex, disease duration, initiation group, and DAS28-CRP as covariates Protocolized models, n: PDQ score $<13 ; n=51$, PDQ score $13-18 ; n=18$, PDQ score $>18 ; n=6$

In contrast, DAS28-CRP at baseline was a significant predictor $(p<0.01)$ of change in the dependent variable (DAS28-CRP, RAMRIS synovitis score, VAS pain) in all adjusted models. Furthermore, RAMRIS synovitis predicted DAS28-CRP change in the unadjusted model ( $p$ $=0.01$ ), and RAMRIS synovitis change both in the unadjusted and the adjusted model $(p \leq 0.02)$. The impact of RAMRIS synovitis score on clinical change is not well documented [38, 39]. Although our group previously has found correlations between RAMRIS synovitis score and DAS28-CRP in another cohort of RA patients (in review), this may be the first study to indicate a possible prognostic value of RAMRIS synovitis score in relation to DAS28CRP change within 4 months. The clinical consistency and relevance of this finding needs further investigation.

Overall the DCE-MRI analyses did not add further information to conventional MRI.

The unexpected behavior of the PDQ may lead to speculations about whether the presents finding are due to the PDQ not being valid in this population. However, the PDQ has, in at least three cross-sectional studies [13, $14,36]$, indicated involvement of non-nociceptive pain mechanisms in subgroups of RA patients. Although we failed to demonstrate a prognostic value of the PDQ, the implications may still be that in patients with clear indication of non-nociceptive pain mechanisms treatment strategies should involve management of chronic pain, including treatment with classes of medication that target central pain mechanisms [6, 7, 40-43], and not only focus on medical treatment of the underlying disease.

The main strength of this study was the prospective design with a rigorous protocol and a prespecified analysis plan. Furthermore, the sample of RA patients was heterogeneous including patients from outpatient clinics and private rheumatologists in the Copenhagen area with a broad spectrum of disease severity. Thus, on one hand this heterogeneity gave the results potential for generalizable interpretation, on the other hand, it was the main limitation in relation to pain profiling, i.e. the constitution of the sample with notably the subgroup of patients with a high baseline PDQ score was small $(n=12)$.

Inflammation was not only assessed clinically but also by MRI of one hand to ensure objectivity. Assessing one hand as a target joint area to reflect general inflammation is a common procedure but can be a limitation to the regression analysis as it involves a risk of misestimating the inflammatory load especially in patients with a non-typical RA presentation. However, it has not yet been clarified how many joints that should be included in a prognostic image analysis to reflect overall inflammatory load.

The study was limited by the framework of the PDQ; the original development for classification of neuropathic pain among patients with various chronic pain conditions, the algorithm for cutoff points also being validated in this sample and the lack of a clinical 'gold standard' assessing augmented central pain mechanisms, against which the PDQ preferably should be tested. However, patients with augmented central pain mechanisms express the same pain features as patients with neuropathic pain, though in a generalized pattern [12, 44-46]. In our opinion this sensitive but not specific feature of the questionnaire, association to tender point count and sign of central sensitization in quantitative sensory testing and functional MRI findings within other chronic pain conditions vouch for the use of the PDQ as indicator of augmented central pain processing [10-12, 47-49].

The relatively short follow-up time of 4 months is a limitation that may have reduced the treatment response for some patients. Further, the baseline visits including MRI could be up to 3 weeks after starting therapy for patients initiating a csDMARD and up to 1 week for patients initiating a bDMARD, which also may have reduced the observed treatment change from baseline to follow-up, particularly on DCE-MRI that is known to be very sensitive to fast inflammatory changes $[31,35,50,51]$. Finally, a direct pain modulating effect of anti-tumor necrosis factor (TNF)-alpha treatment is indicated by the literature [52], and it could therefore be speculated that in some 
patients treated with anti-TNF blocker the allowed delay in baseline assessment may have influenced nociceptive signaling and thereby the study findings.

\section{Conclusions}

In this study, higher numerically changes of inflammatory and patient-reported outcomes, and change of PDQ classification-group at follow-up were observed in the group of patients with a high PDQ score at baseline, which may indicate that inflammatory pain can lead to pain hypersensitivity of a reversible character. Interestingly, overall reduced change in variables was found in patients in the intermediate PDQ classification group with unclear pain mechanism indicating irreversible pain mechanisms. In contrast to our hypothesis, a high PDQ score had no prognostic value in relation to treatment outcome specified as change in DAS28-CRP, RAMRIS score, DCE-MRI, or VAS pain 4 months after initiation or escalation of medical therapy, however, it was found that the RAMRIS synovitis score may have prognostic value in relation to DAS28-CRP response. Further largescale studies are needed to clarify the prognostic value of the PDQ.

\section{Additional files}

Additional file 1: Table showing inter- and intra-reader reliability as intraclass correlation coefficient (ICC) for the DCE-MRI variables. (DOCX $16 \mathrm{~kb}$ )

Additional file 2: Table of multivariable regression models including DCE-MRI variables examining change across PDQ categories expressed as least squares means $(95 \% \mathrm{Cl})$. (DOCX $16 \mathrm{~kb})$

\section{Abbreviations}

ANCOVA: Analysis of covariance; Anti-CCP: Anti-cyclic citrullinated peptide; CRP: C-reactive protein; DAS28-CRP: Disease activity score 28 joints - Creactive protein; DCE-MRI: Dynamic contrast- enhanced magnetic resonance imaging; DMARD: Disease-modifying antirheumatic drug; FM: Fibromyalgia; GAD-10: Generalized anxiety disorder assessment; GH: Global health; HAQ: Health-assessment questionnaire; IRE: Initial rate of enhancement; IQR: Interquartile range; MCP: Metacarpophalangeal; MCS: Mental component score; MDI: Major depression inventory; ME: Maximum enhancement; MRI: Magnetic resonance imaging; MTX: Methotrexate; Nvoxel: Number of enhancing voxel; PCS: Physical component score; PDQ: painDETECT questionnaire; PRO: Patient-reported outcome; RA: Rheumatoid arthritis; RAMRIS: Rheumatoid arthritis magnetic resonance imaging score; RF: Rheumatoid factor; ROI: Region of interest; SF-36: 36-item short form health survey; SJC: Swollen joint count; TJC: Tender joint count; TNF: Tumor necrosis factor; VAS: Visual analogue scale

\section{Acknowledgements}

The authors wish to thank Jack Olsen, Jette Nielsen, and Salomea Hirschorn for assisting with the data acquisition, Christian Cato Holm for data management and Robert C. Riis for sharing his DCE-MRI expertise. We also wish to thank the Departments of Rheumatology at Bispebjerg and Frederiksberg Hospital, Herlev and Gentofte Hospital, and Køge Hospital for assisting with the recruitment of participants. Finally, we wish to thank the Departments of Radiology at Bispebjerg and Frederiksberg Hospital for carrying out the MRI scanning.

\section{Funding}

The study was supported by grants from The Oak Foundation, Selsbjerg Holding, The Danish Rheumatism Association, Minister Erna Hamiltons Legat for Videnskab og Kunst, Axel Muusfeldts Fond, Dagmar Marshalls Fond, Region Hovedstadens Forskningsfond, and Bjarne Jensens Fond.

\section{Availability of data and materials}

The datasets used and/or analyzed during the current study are available from the corresponding author on reasonable request.

\section{Authors' contributions}

All authors participated in the design of the study. SRM, AWC and MB also participated in the data acquisition. All authors participated in drafting the manuscript and have approved it in its final form.

\section{Ethics approval and consent to participate}

This manuscript reports data from clinical research. The study was designed as a clinical prospective cohort study. It was approved by the Regional Ethics Committee of the Capital of Denmark April 18 2013; identification number H-3-2013-049

All participants had to sign a consent form.

\section{Competing interests}

MB reports personal fees from Image Analysis, LTD, London, UK, outside the submitted work. The remaining authors have no competing interests to declare.

\section{Publisher's Note}

Springer Nature remains neutral with regard to jurisdictional claims in published maps and institutional affiliations.

\section{Author details}

'The Parker Institute, Copenhagen University Hospital, Bispebjerg and Frederiksberg, 2000 Frederiksberg, Copenhagen, Denmark. ${ }^{2}$ Department of Rheumatology, Copenhagen University Hospital, Bispebjerg and Frederiksberg, Copenhagen, Denmark. ${ }^{3}$ Department of Rheumatology, Copenhagen University Hospital, Gentofte and Herlev, Hellerup, Denmark. ${ }^{4}$ Department of Radiology, Copenhagen University Hospital, Bispebjerg and Frederiksberg, Copenhagen, Denmark.

Received: 1 November 2017 Accepted: 27 March 2018

Published online: 30 May 2018

\section{References}

1. Schaible HG, von Banchet GS, Boettger MK, Brauer R, Gajda M, Richter F, et al. The role of proinflammatory cytokines in the generation and maintenance of joint pain. Ann N Y Acad Sci. 2010;1193:60-9.

2. Smolen JS, Landewe R, Breedveld FC, Buch M, Burmester G, Dougados M, et al. EULAR recommendations for the management of rheumatoid arthritis with synthetic and biological disease-modifying antirheumatic drugs: 2013 update. Ann Rheum Dis. 2014;73(3):492-509.

3. Meeus M, Vervisch S, De Clerck LS, Moorkens G, Hans G, Nijs J. Central sensitization in patients with rheumatoid arthritis: a systematic literature review. Semin Arthritis Rheum. 2012;41(4):556-67.

4. Lee YC, Cui J, Lu B, Frits ML, lannaccone CK, Shadick NA, et al. Pain persists in DAS28 rheumatoid arthritis remission but not in ACR/EULAR remission: a longitudinal observational study. Arthritis Res Ther. 2011;13(3):R83.

5. Wolfe F, Michaud K. Severe rheumatoid arthritis (RA), worse outcomes, comorbid illness, and sociodemographic disadvantage characterize ra patients with fibromyalgia. J Rheumatol. 2004;31(4):695-700.

6. Lee YC, Nassikas NJ, Clauw DJ. The role of the central nervous system in the generation and maintenance of chronic pain in rheumatoid arthritis, osteoarthritis and fibromyalgia. Arthritis Res Ther. 2011;13(2):211.

7. Woolf $\mathrm{CJ}$. Central sensitization: implications for the diagnosis and treatment of pain. Pain. 2011;152(3 Suppl):S2-15.

8. Aletaha D, Neogi T, Silman AJ, Funovits J, Felson DT, Bingham CO III, et al. 2010 Rheumatoid arthritis classification criteria: an American College of Rheumatology/European League Against Rheumatism collaborative initiative. Arthritis Rheum. 2010;62(9):2569-81.

9. Freynhagen R, Baron R, Gockel U, Tolle TR. painDETECT: a new screening questionnaire to identify neuropathic components in patients with back pain. Curr Med Res Opin. 2006;22(10):1911-20. 
10. Amris K, Jespersen A, Bliddal H. Self-reported somatosensory symptoms of neuropathic pain in fibromyalgia and chronic widespread pain correlate with tender point count and pressure-pain thresholds. Pain. 2010;151(3):664-9.

11. Gwilym SE, Keltner JR, Warnaby CE, Carr AJ, Chizh B, Chessell I, et al. Psychophysical and functional imaging evidence supporting the presence of central sensitization in a cohort of osteoarthritis patients. Arthritis Rheum. 2009;61(9):1226-34

12. Koroschetz J, Rehm SE, Gockel U, Brosz M, Freynhagen R, Tolle TR, et al. Fibromyalgia and neuropathic pain-differences and similarities. A comparison of 3057 patients with diabetic painful neuropathy and fibromyalgia. BMC Neurol. 2011;11:55.

13. Ahmed S, Magan T, Vargas M, Harrison A, Sofat N. Use of the painDETECT tool in rheumatoid arthritis suggests neuropathic and sensization components in pain reporting. J Pain Res. 2014;7:579-88.

14. Koop SM, Ten Klooster PM, Vonkeman HE, Steunebrink LM, van de Laar MA. Neuropathic-like pain features and cross-sectional associations in rheumatoid arthritis. Arthritis Res Ther. 2015;17(1):237.

15. Wu Q, Inman RD, Davis KD. Neuropathic pain in ankylosing spondylitis - a psychophysics and brain imaging study. Arthritis Rheum. 2013;65(6):1494-503.

16. Ostergaard M, Peterfy C, Conaghan P, McQueen F, Bird P, Ejbjerg B, et al. OMERACT Rheumatoid Arthritis Magnetic Resonance Imaging Studies. Core set of MRI acquisitions, joint pathology definitions, and the OMERACT RA-MRI scoring system. J Rheumatol. 2003;30(6):1385-6.

17. Axelsen MB, Stoltenberg M, Poggenborg RP, Kubassova O, Boesen M, Bliddal $\mathrm{H}$, et al. Dynamic gadolinium-enhanced magnetic resonance imaging allows accurate assessment of the synovial inflammatory activity in rheumatoid arthritis knee joints: a comparison with synovial histology. Scand J Rheumatol. 2012;41(2):89-94.

18. Ostergaard M, Stoltenberg M, Lovgreen-Nielsen P, Volck B, Jensen $\mathrm{CH}_{\text {, }}$ Lorenzen I. Magnetic resonance imaging-determined synovial membrane and joint effusion volumes in rheumatoid arthritis and osteoarthritis: comparison with the macroscopic and microscopic appearance of the synovium. Arthritis Rheum. 1997;40(10):1856-67.

19. Christensen AW, Rifbjerg-Madsen S, Christensen R, Amris K, Taylor PC, Locht $H_{\text {, }}$ et al. Temporal summation of pain and ultrasound Doppler activity as predictors of treatment response in patients with rheumatoid arthritis: protocol for the Frederiksberg hospitals Rheumatoid Arthritis, pain assessment and Medical Evaluation (FRAME-cohort) study. BMJ Open. 2014;4(1):e004313.

20. Rifbjerg-Madsen S, Christensen AW, Boesen M, Christensen R, DanneskioldSamsoe B, Bliddal H, et al. Can the painDETECT Questionnaire score and MRI help predict treatment outcome in rheumatoid arthritis: protocol for the Frederiksberg hospital's Rheumatoid Arthritis, pain assessment and Medical Evaluation (FRAME-cohort) study. BMJ Open. 2014;4(11):e006058.

21. Arnett FC, Edworthy SM, Bloch DA, McShane DJ, Fries JF, Cooper NS, et al. The American Rheumatism Association 1987 revised criteria for the classification of rheumatoid arthritis. Arthritis Rheum. 1988;31(3):315-24.

22. Rifbjerg-Madsen S, Waehrens EE, Danneskiold-Samsoe B, Amris K. Psychometric properties of the painDETECT questionnaire in rheumatoid arthritis, psoriatic arthritis and spondyloarthritis: Rasch analysis and test-retest reliability. Health Qual Life Outcomes. 2017;15(1):110.

23. Gudbergsen $H$, Bartels EM, Krusager $P$, Waehrens EE, Christensen $R$, Danneskiold-Samsoe B, et al. Test-retest of computerized health status questionnaires frequently used in the monitoring of knee osteoarthritis: a randomized crossover trial. BMC Musculoskelet Disord. 2011;12:190.

24. Thorsen H, Hansen TM, MCKenna SP, Sorensen SF, Whalley D. Adaptation into Danish of the Stanford Health Assessment Questionnaire (HAQ) and the Rheumatoid Arthritis Quality of Life Scale (RAQoL). Scand J Rheumatol. 2001;30(2):103-9.

25. Bjorner JB, Thunedborg K, Kristensen TS, Modvig J, Bech P. The Danish SF-36 Health Survey: translation and preliminary validity studies. J Clin Epidemiol. 1998;51(11):991-9.

26. Bech $\mathrm{P}$, Kastrup M, Rafaelsen OJ. Mini-compendium of rating scales for states of anxiety depression mania schizophrenia with corresponding DSM-III syndromes. Acta Psychiatr Scand Suppl. 1986;326:1-37.

27. Bech P, Rasmussen NA, Olsen LR, Noerholm V, Abildgaard W. The sensitivity and specificity of the Major Depression Inventory, using the Present State Examination as the index of diagnostic validity. J Affect Disord. 2001;66(2-3):159-64

28. Ejbjerg B, McQueen F, Lassere M, Haavardsholm E, Conaghan P, O'Connor P, et al. The EULAR-OMERACT rheumatoid arthritis MRI reference image atlas: the wrist joint. Ann Rheum Dis. 2005;64(Suppl 1):i23-47.
29. Haavardsholm EA, Ostergaard M, Ejbjerg BJ, Kvan NP, Uhlig TA, Lilleas FG, et al. Reliability and sensitivity to change of the OMERACT rheumatoid arthritis magnetic resonance imaging score in a multireader, longitudinal setting. Arthritis Rheum. 2005;52(12):3860-7.

30. Boesen M, Kubassova O, Parodi M, Bliddal H, Innocenti S, Garlaschi G, et al. Comparison of the manual and computer-aided techniques for evaluation of wrist synovitis using dynamic contrast-enhanced MRI on a dedicated scanner. Eur J Radiol. 2011;77(2):202-6.

31. Axelsen MB, Poggenborg RP, Stoltenberg M, Kubassova O, Boesen M, Horslev-Petersen $\mathrm{K}$, et al. Reliability and responsiveness of dynamic contrastenhanced magnetic resonance imaging in rheumatoid arthritis. Scand J Rheumatol. 2013;42(2):115-22.

32. Ballegaard $C$, Riis RG, Bliddal $H$, Christensen R, Henriksen M, Bartels EM, et al. Knee pain and inflammation in the infrapatellar fat pad estimated by conventional and dynamic contrast-enhanced magnetic resonance imaging in obese patients with osteoarthritis: a cross-sectional study. Osteoarthr Cartil. 2014;22(7):933-40

33. Bandak E, Boesen M, Bliddal $H$, Riis RG, Gudbergsen $H$, Henriksen M. Associations between muscle perfusion and symptoms in knee osteoarthritis: a cross sectional study. Osteoarthr Cartil. 2015;23(10):1721-7.

34. Boesen M, Kubassova O, Bouert R, Axelsen MB, Ostergaard M, Cimmino MA, et al. Correlation between computer-aided dynamic gadolinium-enhanced MRI assessment of inflammation and semi-quantitative synovitis and bone marrow oedema scores of the wrist in patients with rheumatoid arthritis-a cohort study. Rheumatology (Oxford). 2012;51(1):134-43.

35. Cimmino MA, Parodi M, Zampogna G, Boesen M, Kubassova O, Barbieri F, et al. Dynamic contrast-enhanced, extremity-dedicated MRI identifies synovitis changes in the follow-up of rheumatoid arthritis patients treated with rituximab. Clin Exp Rheumatol. 2014;32(5):647-52.

36. Christensen AW, Rifbjerg-Madsen S, Christensen R, Dreyer L, Tillingsoe H, Seven $S$, et al. Non-nociceptive pain in rheumatoid arthritis is frequent and affects disease activity estimation: cross-sectional data from the FRAME study. Scand J Rheumatol. 2016;18:1-9.

37. Christensen AW, Rifbjerg-Madsen S, Christensen R, Dreyer L, Boesen M, Ellegaard K, et al. Ultrasound Doppler but not temporal summation of pain predicts DAS28 response in rheumatoid arthritis: a prospective cohort study. Rheumatology (Oxford). 2016;55(6):1091-8.

38. Ostergaard M, Moller-Bisgaard S. Optimal use of MRI in clinical trials, clinical care and clinical registries of patients with rheumatoid arthritis. Clin Exp Rheumatol. 2014;32(5 Suppl 85):S-22.

39. Suter LG, Fraenkel L, Braithwaite RS. Role of magnetic resonance imaging in the diagnosis and prognosis of rheumatoid arthritis. Arthritis Care Res (Hoboken ). 2011;63(5):675-88.

40. Brendbekken R, Eriksen HR, Grasdal A, Harris A, Hagen EM, Tangen T. Return to work in patients with chronic musculoskeletal pain: multidisciplinary intervention versus brief intervention: a randomized clinical trial. J Occup Rehabil. 2017;27(1):82-91.

41. Tse MM, Yeung SS, Lee PH, Ng SS. Effects of a peer-led pain management program for nursing home residents with chronic pain: a pilot study. Pain Med. 2016;18.

42. Amris K, Luta G, Christensen R, Danneskiold-Samsoe B, Bliddal H, Waehrens EE. Predictors of improvement in observed functional ability in patients with fibromyalgia as an outcome of rehabilitation. J Rehabil Med. 2016;48(1):65-71.

43. Amris K, Waehrens EE, Christensen R, Bliddal H, Danneskiold-Samsoe B. Interdisciplinary rehabilitation of patients with chronic widespread pain: primary endpoint of the randomized, nonblinded, parallel-group IMPROvE trial. Pain. 2014;155(7):1356-64.

44. Baron R, Binder A, Wasner G. Neuropathic pain: diagnosis, pathophysiological mechanisms, and treatment. Lancet Neurol. 2010;9(8):807-19.

45. Bennett MI, Attal N, Backonja MM, Baron R, Bouhassira D, Freynhagen R, et al. Using screening tools to identify neuropathic pain. Pain. 2007; 127(3):199-203.

46. Rehm SE, Koroschetz J, Gockel U, Brosz M, Freynhagen R, Tolle TR, et al. A cross-sectional survey of 3035 patients with fibromyalgia: subgroups of patients with typical comorbidities and sensory symptom profiles. Rheumatology (Oxford). 2010;49(6):1146-52.

47. Hochman JR, Gagliese L, Davis AM, Hawker GA. Neuropathic pain symptoms in a community knee OA cohort. Osteoarthr Cartil. 2011;19(6):647-54.

48. Hochman JR, Davis AM, Elkayam J, Gagliese L, Hawker GA. Neuropathic pain symptoms on the modified painDETECT correlate with signs of central sensitization in knee osteoarthritis. Osteoarthr Cartil. 2013;21(9):1236-42. 
49. Moreton BJ, Tew V, das NR WM, Walsh DA, Lincoln NB. Pain phenotype in patients with knee osteoarthritis: classification and measurement properties of painDETECT and self-report Leeds assessment of neuropathic symptoms and signs scale in a cross-sectional study. Arthritis Care Res (Hoboken ). 2015;67(4):519-28.

50. Boesen M, Kubassova O, Cimmino MA, Ostergaard M, Taylor P, Danneskiold-Samsoe B, et al. Dynamic contrast-enhanced MRI can monitor the very early inflammatory treatment response upon intraarticular steroid injection in the knee joint: a case report with review of the literature. Arthritis. 2011;2011:578252.

51. Conaghan PG, Ostergaard M, Bowes MA, Wu C, Fuerst T, van der Heijde $D$, et al. Comparing the effects of tofacitinib, methotrexate and the combination, on bone marrow oedema, synovitis and bone erosion in methotrexate-naive, early active rheumatoid arthritis: results of an exploratory randomised MRI study incorporating semiquantitative and quantitative techniques. Ann Rheum Dis. 2016;75(6):1024-33.

52. Hess A, Axmann R, Rech J, Finzel S, Heindl C, Kreitz S, et al. Blockade of TNF-alpha rapidly inhibits pain responses in the central nervous system. Proc Natl Acad Sci U S A. 2011;108(9):3731-6.

\section{Submit your next manuscript to BioMed Central} and we will help you at every step:

- We accept pre-submission inquiries

- Our selector tool helps you to find the most relevant journal

- We provide round the clock customer support

- Convenient online submission

- Thorough peer review

- Inclusion in PubMed and all major indexing services

- Maximum visibility for your research

Submit your manuscript at www.biomedcentral.com/submit 